\title{
Control of mixing step in the bread production with weak wheat flour and sourdough
}

\author{
Alessandro Parenti, ${ }^{1}$ Lorenzo Guerrini, ${ }^{1}$ Lisa Granchi, ${ }^{1}$ Manuel Venturi, ${ }^{1}$ \\ Stefano Benedettelli, ${ }^{2}$ Fabrizio Nistri ${ }^{3}$
}

\author{
${ }^{1}$ Dipartimento di Gestione dei Sistemi Agrari, Alimentari e Forestali, Università di Firenze, Italy; \\ ${ }^{2}$ Dipartimento di Scienze delle Produzioni Agroalimentari e dell'Ambiente, Università di Firenze, \\ Italy; ${ }^{3}$ Tecnologie per l'arte bianca di Fabrizio Nistri \& C. Sas, Prato, Italy
}

\begin{abstract}
Recently, several old Italian grain varieties have been reinstated, and the market seems to reward the breads made with these flours. Among such varieties, cultivar Verna appears to be interesting because the regular consumption of bread obtained by this variety and sourdough provides beneficial effects on human health such as the improving of the lipid, inflammatory, and hemorheological profiles. However, flours derived from Verna shows low technological performances. For example, the $W$ value of these flours, obtained with alveoghraphic tests and considered as the commercial standard for the flour "strength" evaluation, is largely inferior than the $\mathrm{W}$ values of the commercial flour blends currently used in the bread making process. Moreover, the W values broadly change among the batches of Verna flours, whereas, usually, commercial blends are provided to bakeries with standard technological properties. Hence, these properties of Verna flour could lead to developed or overworked doughs and therefore to breads of worse quality. In addition, the previous mentioned large variability of flours from Verna can affect also the sourdough microbiota. For these reasons the composition and activity of the sourdough microorganisms should be controlled while the mixing process should be able to adapt to the different flour properties. Some works, in literature, report
\end{abstract}

Correspondence: Lorenzo Guerrini, Dipartimento di Gestione dei Sistemi Agrari, Alimentari e Forestali, Università degli Studi di Firenze, Piazzale delle Cascine 15, 50144, Firenze, Italy.

Tel. +39.055.3288352 - Fax: +39.055.3288316.

E-mail: lorenzo.guerrini@unifi.it

Key words: baking, sourdough, mixing, old wheat varieties, Verna

Contributions: the authors contributed equally.

Conflict of interests: the authors declare no potential conflict of interests.

Funding: the work was supported by Panificio Menchetti Pietro di Santi e Figli snc, Marciano della Chiana-AR, Italy

(C) Copyright A. Parenti et al., 2013

Licensee PAGEPress, Italy

Journal of Agricultural Engineering 2013; XLIV(s2):e65

doi:10.4081/jae.2013.s2.e65

This article is distributed under the terms of the Creative Commons Attribution Noncommercial License (by-nc 3.0) which permits any noncommercial use, distribution, and reproduction in any medium, provided the original author(s) and source are credited. that monitoring the electrical consumption could provide useful information about the dough rheology, and this could be used to monitor the mixing step. In the present work the effect of different mixing times are evaluated on breads made with Verna flour type 2 leavened with sourdough. Tests were carried out at industrial scale in two different days. During the tests the electric consumption was monitored to highlight some features suitable for the mixing phase control. The breads were evaluated in terms of loaf volume measurement, crumb image analysis and losses of moisture content during storage. The results show that the composition of the sourdough microbiota and the mixing time affects the produced bread, especially when it is baked with low technological performance flours. Bread baked with an appropriate mixing time shows higher loaf volumes and lower water losses during storage.

\section{Introduction}

During the last 50 years, wheat breeding strategies were direct to the yield production improvement, to the kernel protein content increase, and to select plant with high response to chemical fertilization input (Sofi et al., 2010). Gradually, in this scenario, old varieties are less and less suitable in agricultural production, and they were confined to germoplasm collections and seed servers. Nowadays, however, this trend seems to be reversed. In fact, in Tuscany, breads made with type 2 flours from these varieties and sourdough as leavening agent restart to spread, and the market seems to reward these breads. This could be due to the increased consumer attention to healthy and functional foods. Particularly, a study on regular consumption of bread made with Verna flour and sourdough shows beneficial in several aspects associated to the consumer health. Indeed, the lipid, inflammatory, and hemorheological profiles results improved by Verna bread regular consumption (Sofi et al., 2010). In terms of bread productive process, the spread of old varieties, and the use of sourdough, raise up some issues. Currently, bread makers work blend of flours, with standard technological characteristics, and perhaps adopt a standard recipe, with well-defined working conditions, i.e. temperature, water content, absence/presence of salt and surfactant, amount of mixing work input and baker's yeast as leavening agent. These conditions are widely considered important to the success of the productive process (Campos et al, 1997; Anderssen et al., 1997). In bakery, the commercial standard for the flour strength evaluation, is the alveographic test; with the reference value of the parameter "W", which summarizes technological flours performance. In normal bread-making process, blends shows constant $\mathrm{W}$ values, so a standard work-input could be provided in the mixing phase, guaranteeing the final bread acceptability. On the other hand, with old varieties flours, the $\mathrm{W}$ is largely variable among the batches and a standard recipe could not be adopted 
anymore. Also, the $\mathrm{W}$ value of these flours is lower than those of the commonly used flour. For Verna, to provide an example of the working difficulties in bakery of these flours, $W$ value of about 100 could be found in literature (Parenti et al., 2012), while Quaglia (1984) identified wheat with W lower than 110 as "not for bread-making use". The various flour characteristics and process parameters affect also the performances of the sourdough that is used in the leaving step during the bread making in order to improve rheological, organoleptic and nutritional properties of the final products (Arendt et al., 2007). Indeed, the sourdough microbiota, including lactic acid bacteria mostly of the genus Lactobacillus and yeast species belonging to genera Candida and Saccharomyces, are known to be dependent on some process parameters such as dough yield (water activity), addition of salt, number of propagation steps, and fermentation time (De Vuys and Neysens, 2005). On the contrary, surveys on the possible effect of the dough mixing time on the microbial ecology of the sourdough fermentation are lacking.

These issues point out the importance to investigate the effect of mixing work input amount from both technological and microbiological points of view.

The mixing work input provides to flour and water the energy allowing the development of a protein matrix, including starch granules, and giving dough the peculiar viscoelastic behaviour (gluten network). During the mixing step, after the complete flour hydration, the adjunctive energy is used for stretching and orienting the protein electronegative groups, to allow the formation of covalent bounds among cysteine residues, and other kinds of bounds, such as dipolar, ionic, hydrogen and Van der Walls forces. A well-hydrated dough, with insufficient mechanic deformation is named undeveloped dough and present worse rheological and baking properties than a correct developed one (Campos et al., 1997). Even overworked dough shows mediocre technological properties, with decreased resistance and extensibility before the breaking (Gras, 2000). Hence, to obtain quality breads (in rheological terms) is important to provide a well-defined mixing work input. In the present work, a monitoring of the effects at industrial scale of different work input on breads has been carried out.

\section{Materials and methods}

Trials were carried out at Panificio Menchetti Pietro di Santi e Figli snc (Marciano della Chiana-AR, Italy), during two tests days. The used mixer was a twin arm type with $80 \mathrm{~kg}$ of maximum capacity, filled with $60 \mathrm{~kg}$ of dough, working at $30 \mathrm{rpm}$ of mixing rate. The adopted proportion among ingredient was: $30 \mathrm{~kg}$ Verna type 2 flour (Table 1), $15 \mathrm{~kg}$ of sourdough, $15 \mathrm{~kg}$ of water.

In the two tests the effect of mixing were evaluated after 12,17 , and 22 minutes in the first one (PM1), and after 17, 22, and 27 in the last

Table 1. Flour technological characterization.

\begin{tabular}{lccc}
\multicolumn{2}{c}{ Rheological characteristics } & Chemical characteristics (\%) \\
W & $100-120^{*} 10^{-4} \mathrm{~J}$ & Humidity & 11 \\
P/L & 1.09 & Total Protein & 11 \\
\hline Falling Number & $310-330$ & Insoluble Protein & 10 \\
Stability & $>6$ & Ashes & 1.50 \\
\hline Water absorption & $>45 \%$ & Cellulose & $>1.6$ \\
Volume & $>550 \mathrm{~m} / 100 \mathrm{~g}$ & Lipids & 0.50 \\
\hline
\end{tabular}

one (PM2). At the chosen moments three samples of dough per each time were collected and put in mould. Afterwards, the samples were put in leavening cell at $30^{\circ} \mathrm{C}$ and $75 \%$ of relative humidity. The proofing trend was monitored with $\mathrm{pH}$ measures performed every 30 minutes, and putting another sample in a graduate cylinder. Microbiological analysis of different samples were performed at the beginning and at the end of the fermentation. The proofing ended at $3.5 \mathrm{of} \mathrm{pH}$ value; this happened before 3 and 2.5 hours respectively, in the first, and in the second test. Then, samples were baked. The steam oven annular tubes (Spighi, Italy), was set up to $240^{\circ} \mathrm{C}$ and, after the bread introduction the heating system was turned off. The chosen baking time was 55 minutes.

pH measurement: $\mathrm{pH}$ was measured with a Crison pHmeter (mod GLP 21), after dissolving a small dough sample of about $10 \mathrm{~g}$ in distilled water.

Microbiological analysis: Dough samplings and platings were carried out at the manufacture soon after the mixing step and at the end of fermentation. Lactic acid bacteria, plated on maltose-MRS agar integrated with pimaricin $(50 \mathrm{mg} / \mathrm{L})$ at $\mathrm{pH} 5.6$, were counted after incubation for $48-72 \mathrm{~h}$ at $30^{\circ} \mathrm{C}$ under anaerobic conditions. Yeasts, plated on WL agar (Oxoid Ltd, Basingstoke, Hampshire, UK) containing sodium propionate $(2 \mathrm{~g} / \mathrm{L})$ and streptomycin $(30 \mathrm{mg} / \mathrm{L})$, were counted after incubation for $48 \mathrm{~h}$ at $30^{\circ} \mathrm{C}$ under aerobic conditions. Identification of microbial isolates was attained by molecular methods: PCR-RFLP of ITS region for yeasts and PCR-ARDRA for lactic acid bacteria according to Venturi et al. (2012).

Volume increase monitoring: $500 \mathrm{~g}$ of dough were putted into the leavening cell into a graduated cylinder. Dough height was recorded every 30 minutes.

The results of the different mixing time were evaluated in term of final loaf height, crumb image analysis, and losses of moisture during storage.

Final loaf height: three samples of $1000 \mathrm{~g}$ of dough were collected for each time and put in a mould. The mould was parallelepiped with internal dimension of mm 105x $250 \times 60$ with the upper side open to allow the dough proofing. These moulds, hence, permit to compare different thesis during proofing.

Crumb image analysis: three slices of each loaf were analysed according to the method already described in Fois et al. (2012).

Moisture losses: changes in crumb moisture content were evaluated with a loss on drying method. About $5 \mathrm{~g}$ of crumb were exactly weighted and put in oven overnight at $103^{\circ} \mathrm{C}$. Afterwards, the sample was weighted again, and the water content determined from the difference. The measures were performed after 1,3 , and 7 days since baking.

\section{Results and discussion}

The three different working times return breads with different appearance. During the leavening phase, the different mixing time produces different leavening behaviour (Figure 1A and 1B).

The sample with the lower mixing input (12 minutes), and those with the higher one (27 minutes) showed less volume increase in the proofing phase, than the sample picked at 17 minutes that showed the highest value in both tests.

Such differences couldn't be attributed to microbial populations occurring in dough after the different mixing time and at the end of fermentation. Indeed, in both trials, independently of the mixing and fermentation time, the yeast population, consisting of the species Candida milleri, achieved comparable cell densities (Table 2). In addition, although, soon after the mixing step, bacterial populations decreased progressively with the increase of working time, at the end 


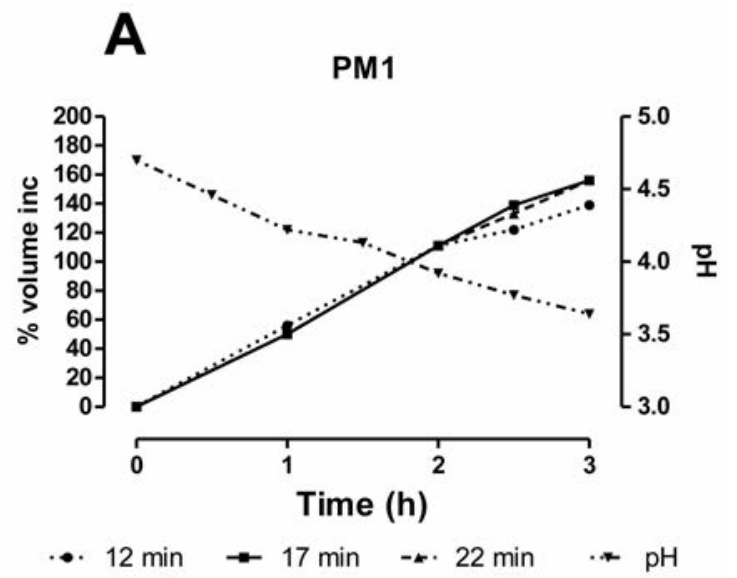

B

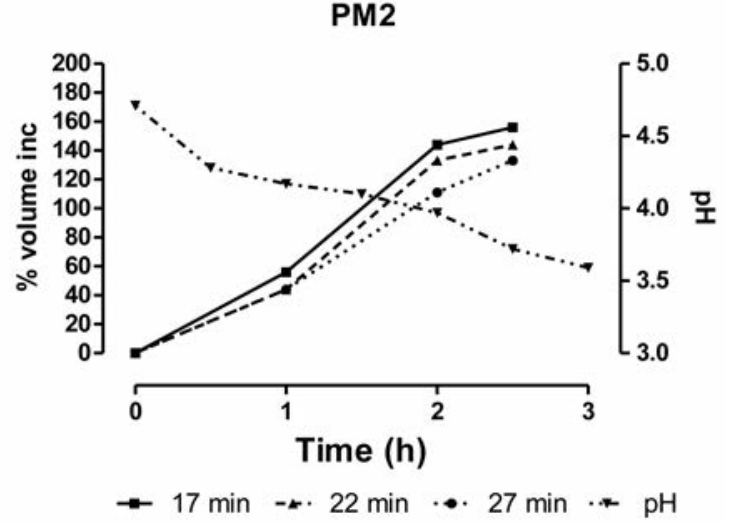

Figure 1. Volume increase (\%) and $\mathrm{pH}$ courses during the fermentation in the two trials; A: PM1; B: PM2.

Table 2. Microbiological counts (CFU/g) and standard deviations (sd) of Lactobacillus sanfranciscensis (Ls) and Candida milleri $(\mathrm{Cm})$ at the beginning (T0) and at the end of the fermentation after 3 (T3) or 2.5 (T2.5) $h$ in the trials PM1 and PM2, respectively. Numbers at pedice after $F$ indicate the minutes of dough mixing time.

\begin{tabular}{lcccc} 
PM1 & Ls & Cm & & \\
CFU/g & sd & CFU/g & sd \\
F $_{12}$ T0 & $2.62 \times 10^{8}$ & $3.32 \times 10^{7}$ & $6.10 \times 10^{6}$ & $5.66 \times 10^{5}$ \\
$\mathrm{~F}_{17} \mathrm{~T} 0$ & $1.28 \times 10^{8}$ & $1.48 \times 10^{7}$ & $4.75 \times 10^{6}$ & $3.54 \times 10^{5}$ \\
\hline $\mathrm{F}_{22} \mathrm{~T} 0$ & $9.85 \times 10^{7}$ & $9.19 \times 10^{6}$ & $4.60 \times 10^{6}$ & $7.07 \times 10^{5}$ \\
$\mathrm{~F}_{12} \mathrm{~T} 3$ & $4.70 \times 10^{8}$ & $2.26 \times 10^{7}$ & $1.89 \times 10^{7}$ & $3.59 \times 10^{5}$ \\
\hline $\mathrm{F}_{17} \mathrm{~T} 3$ & $4.05 \times 10^{8}$ & $2.47 \times 10^{7}$ & $1.24 \times 10^{7}$ & $1.64 \times 10^{5}$ \\
$\mathrm{~F}_{22} \mathrm{~T} 3$ & $9.70 \times 10^{8}$ & $1.13 \times 10^{7}$ & $1.63 \times 10^{7}$ & $3.01 \times 10^{5}$ \\
\hline PM2 & $\mathrm{Ls}$ & $\mathrm{Cm}$ & & \\
& $\mathrm{CFU} / \mathrm{g}$ & sd & $\mathrm{CFU} / \mathrm{g}$ & $\mathrm{sd}$ \\
\hline $\mathrm{F}_{17} \mathrm{~T} 0$ & $2.09 \times 10^{8}$ & $1.13 \times 10^{7}$ & $4.50 \times 10^{6}$ & $5.66 \times 10^{5}$ \\
$\mathrm{~F}_{22} \mathrm{~T} 0$ & $1.53 \times 10^{8}$ & $1.56 \times 10^{7}$ & $3.00 \times 10^{6}$ & $1.41 \times 10^{5}$ \\
\hline $\mathrm{F}_{27} \mathrm{~T} 0$ & $9.85 \times 10^{7}$ & $1.34 \times 10^{7}$ & $4.35 \times 10^{6}$ & $7.78 \times 10^{5}$ \\
$\mathrm{~F}_{17} \mathrm{~T} 2,5$ & $6.05 \times 10^{8}$ & $1.20 \times 10^{7}$ & $8.70 \times 10^{6}$ & $5.46 \times 10^{5}$ \\
\hline $\mathrm{F}_{22} \mathrm{~T} 2,5$ & $8.90 \times 10^{8}$ & $5.66 \times 10^{6}$ & $1.28 \times 10^{7}$ & $1.62 \times 10^{5}$ \\
$\mathrm{~F}_{27} \mathrm{~T} 2,5$ & $1.03 \times 10^{9}$ & $7.78 \times 10^{6}$ & $2.66 \times 10^{7}$ & $6.49 \times 10^{5}$ \\
\hline
\end{tabular}

of fermentation, they didn't show significant differences. This means that the longer was the mixing time the higher was, during the fermentation, the number of generations of Lactobacillus sanfranciscensis, the only bacterial species found. Nevertheless, no relationships between the extent of growth and fermentation performances were established. Anyway, it is pointed out that, as expected, lactic acid bacteria was the predominant population in dough,

The volume increase was only partially reflected in the height of the breads showed in Figures 2A and 2B.

In the first test, the heights among the mixing times are well pronounced, in fact the Tukey post-hoc test shows significant difference $(\mathrm{P}=0.05)$ among the three treatments. On the other hand, in the second test the baking process partially overcome the proofing difference and difference could be found only between 22 and 27 minutes. In the crumb image analysis the holes were divided in three class: $\mathrm{I} \leq 0.05 \mathrm{~mm}^{3}, 0.05$ $\mathrm{mm}^{3} \leq$ II $\leq 3 \mathrm{~mm}^{3}$, III $>3 \mathrm{~mm}^{3}$. Also, the ratio between total and alveolated surface was determined and express in percentage (Table 3 ).

Data didn't show pronounced differences among the mixing time, except for the under mixed dough. In fact, the slice image analysis revealed, in comparison i.e. with the 22 minutes of mixing of the same test, the same percentage of alveolated surface, but larger and irregular holes on the slice. The crumb water content during storage is shown in Figures $3 \mathrm{~A}$ and $3 \mathrm{~B}$.

In this case, differences in bread water retention could be noticed for
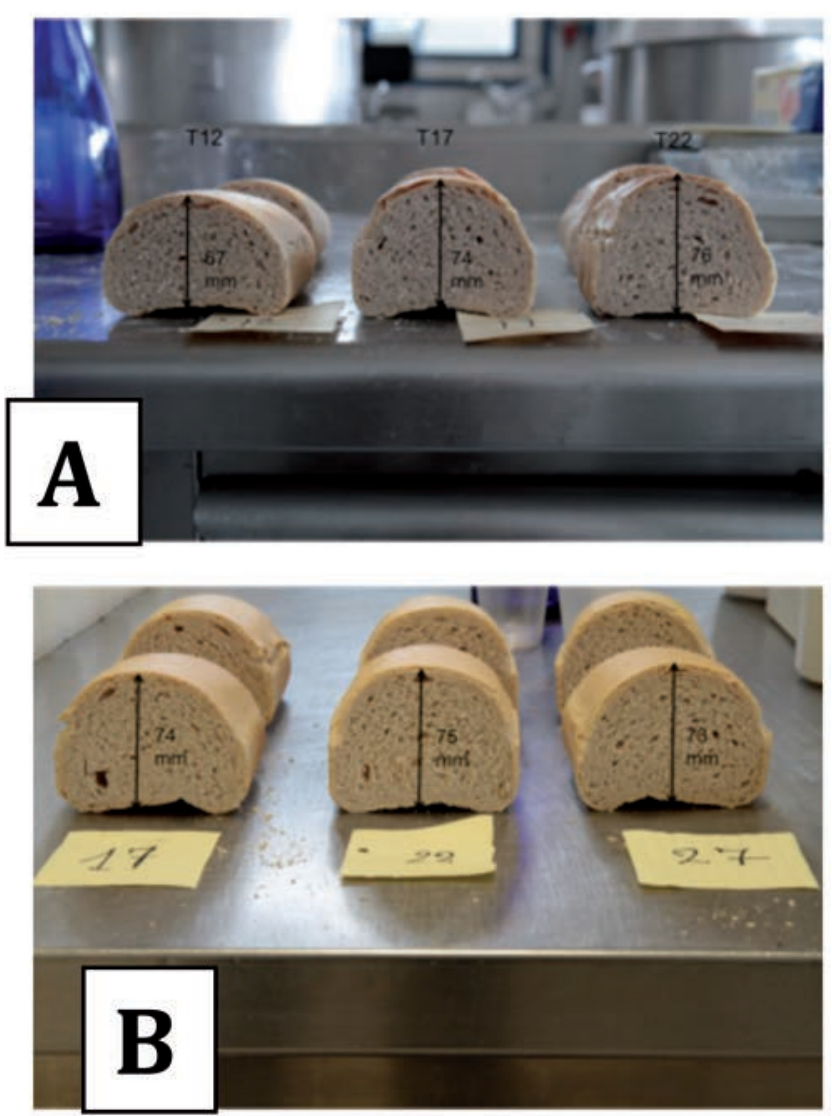

Figure 2. Height of the breads with different mixing times. A: PM1; B: PM2. 
Table 3. Results of crumb image analysis of the loafs in the two trials.

\begin{tabular}{|c|c|c|c|c|c|c|c|}
\hline \multirow[t]{2}{*}{ PM1 } & \multicolumn{3}{|c|}{ Class distribution (\%) } & \multicolumn{3}{|c|}{ Average hole surface $\left(\mathrm{mm}^{3}\right)$ per class } & \multirow[t]{2}{*}{ Alveolated surface (\%) } \\
\hline & I & II & III & I & II & III & \\
\hline $\mathrm{T} 12$ & 67.45 & 21.25 & 11.30 & 0.169 & 1.249 & 8.283 & 29.10 \\
\hline $\mathrm{T} 17$ & 72.55 & 17.90 & 9.55 & 0.174 & 1.298 & 7.859 & 25.05 \\
\hline T22 & 71.55 & 21.20 & 7.25 & 0.176 & 1.091 & 10.654 & 29.10 \\
\hline PM2 & \multicolumn{3}{|c|}{ Class distribution (\%) } & \multicolumn{3}{|c|}{ Average hole surface $\left(\mathrm{mm}^{3}\right)$ per class } & Alveolated surface (\%) \\
\hline $\mathrm{T} 17$ & 71.33 & 22.30 & 6.38 & 0.166 & 1.234 & 7.383 & 31.20 \\
\hline T22 & 73.83 & 19.18 & 6.98 & 0.161 & 1.190 & 7.900 & 27.00 \\
\hline $\mathrm{T} 27$ & 72.50 & 21.03 & 6.48 & 0.161 & 1.181 & 10.809 & 31.70 \\
\hline
\end{tabular}
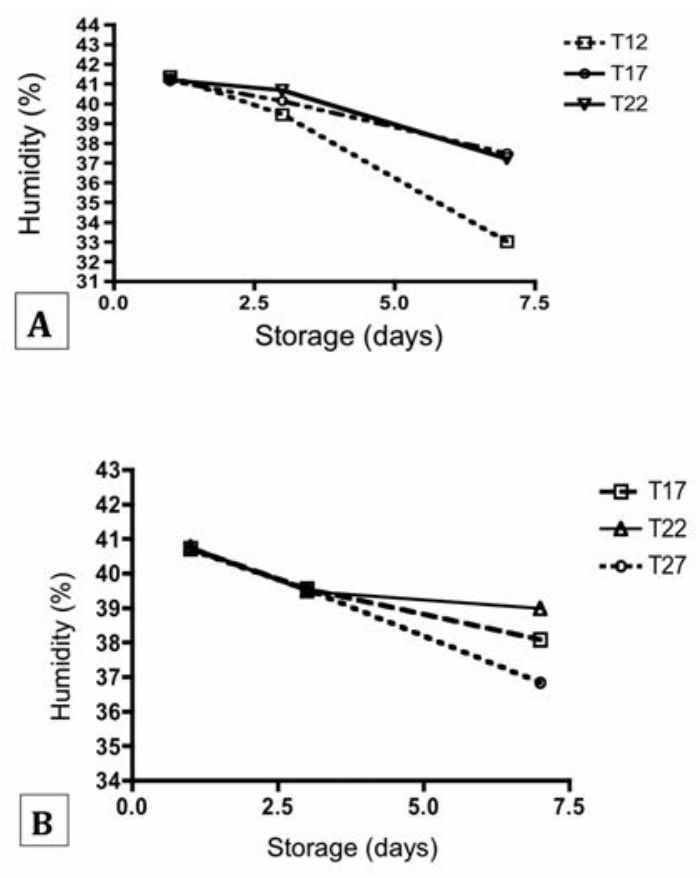

Figure 3. Humidity trend during storage of the different breads . A: PM1; B: PM2.

breads mixed for a different time. Particularly, in the first test, there is a significant difference between the 12 minutes treatment, and the others. On the other hand, in the second test, there are differences among the three times. These changes in moisture are less pronounced during the first days of storage, and became significant after a week.

\section{Conclusions}

In the present work some effect of the mixing time on the produced Verna breads could be noticed. Particularly, those effects appear more pronounced during the proofing phase, and could be revealed in terms of dough growth and bread final volume. Furthermore, the mixing time affect the humidity retention capacity of the breads during storage. In literature (Zheng et al., 2000; Shehzad et al., 2012), some works pointed out that electrical consumption could be suitable to monitor the dough behaviour during the mixing step. Considering the importance of this step on bread production and the already seen effects of mixing time, further development should be oriented to the use of this parameter to attempt establishing the optimal mixing end.

\section{References}

Anderssen, R. S., Gras, P. W., \& Macritchie, F. 1998. The rate-independence of the mixing of wheat flour dough to peak dough development. J Cereal Sci. 27, 167-177.

Arendt, E. K., Ryan, L. A. M., \& Dal Bello, F. Impact of sourdough on the texture of bread. 2007. Food Microbiol., 24, 165-174.

Campos, D. T., Steffe, J. F., \& Ng, P. K. W. 1997. Rheological behavior of undeveloped and developed wheat dough. Cereal Chem., 489-494.

De Vuyst, L., \& Neysens, P. The sourdough microflora: biodiversity and metabolic interactions. 2005. Trends Food Sci. Tech., 16, 43 - 56.

Fois, S., Fadda, C., Tonelli, R., et al. 2012. Effects of the fermentation process on gas-cell size two-dimensional distribution and rheological characteristics of durum-wheat-based doughs. Food Res. Int., 49(1), 193-200.

Gras, P. 2000. Modelling the developmental rheology of wheat-flour dough using extension tests. J Cereal Sci., 31(1), 1-13.

Parenti, A., Guerrini, L., \& Spugnoli, P. Dough buoyancy to monitor bread production process. In: AgEng2012: proceedings of the international conference of agricultural engineering. 2012, July 8-12.

Shehzad, A., Chiron, H., Della Valle, et al. 2012. Energetical and rheological approaches of wheat flour dough mixing with a spiral mixer. J. Food Eng., 110(1), 60-70.

Sofi, F., Ghiselli, L., Cesari, F., et al. 2010. Effects of short-term consumption of bread obtained by an old italian grain variety on lipid, inflammatory, and hemorheological variables: an intervention study. J. Med Food, 13(3), 1-7.

Quaglia G. 1984. Scienza e tecnologia della panificazione. Chirotti editori. Pinerolo - Italy

Venturi M., Guerrini S., \& Vincenzini M. 2012. Stable and non-competitive association of Saccharomyces cerevisiae, Candida milleri and Lactobacillus sanfranciscensis during manufacture of two traditional sourdough baked goods. Food Microbiol. 31, 107e115.

Zheng, H., Morgenstern, M. P., Campanella, O. H., \& G, L. N. 2000. Rheological properties of dough during mechanical dough development. J Cereal Sci. 32(3), 293-306. 